\title{
Pollen grains in quaternary sediments from the Campos Basin, state of Rio de Janeiro, Brazil: Core BU-91-GL-05
}

\author{
Aline Gonçalves de Freitas ${ }^{1,2,4}$, Marcelo de Araujo Carvalho², \\ Claudia Barbieri Ferreira Mendonça ${ }^{3}$ and Vania Gonçalves-Esteves ${ }^{3}$
}

Received: 16 August, 2012. Accepted: 1 August, 2013

\begin{abstract}
Here, we describe pollen grains extracted from a Pleistocene-Holocene sediment core (BU-91-GL-05; 22 $48^{\prime} 45^{\prime \prime}$; $\left.41^{\circ} 54^{\prime} 13^{\prime \prime} \mathrm{W}\right)$ taken from the Albacora Slope ( $22^{\circ} 48^{\prime} 45^{\prime \prime}$; $\left.41^{\circ} 54^{\prime} 13^{\prime \prime} \mathrm{W}\right)$, located in the Campos Basin of the northern region of the state of Rio de Janeiro, Brazil. The analysis resulted in the identification and morphological description of 46 types of pollen: one of a gymnosperm genus (Podocarpus); and 45 of angiosperm taxa across 27 families-one family of monocotyledons (Poaceae) and 26 families (30 types) of dicotyledons. The most common angiosperm families were Amaranthaceae (Chenopodium, Amaranthus and Gomphrena); Fabaceae (Fabaceae type, Bauhinia, Inga and Canavalia); Malpighiaceae (Tetrapteris, Heteropteris and Peixotoa); Malvaceae (Sida, Abutilon, Hibiscus and Pseudobombax); Rubiaceae (Faramea, Borreria and Psychotria); Asteraceae (Eupatorium and tribe Vernonieae); Bignoniaceae (Bignoniaceae type, Adenocalymma and Tabebuia); and Onagraceae (Fuchsia and Ludwigia). The palynoflora in this study are associated with dense montane and submontane Atlantic Forest, semideciduous forest and restinga (coastal woodland), all of which are present in the state of Rio de Janeiro. Palynological analysis can provide important data about paleovegetation and paleoclimatic changes in the studied area during the Quaternary, specifically in the last 145,000 years.
\end{abstract}

Key words: palynology; pollen descriptions; paleovegetation; Quaternary; Campos Basin

\section{Introduction}

Morphological descriptions and taxonomic identification of quaternary pollen grains form marine sediments can allow inferences to be made about the paleovegetation, paleoclimate and paleoenvironment during the Upper Pleistocene-Holocene in coastal areas of southeastern Brazil. Fossil pollen grains and spores are morphologically similar to those produced by modern plants. Although the palynological records collected to date for southern and southeastern Brazil are relatively specific, these data constitute an important tool for the understanding of vegetation dynamics and paleoclimate, as well as for and paleoenvironmental reconstruction of the Pleistocene-Holocene (Behling et al. 2002; Freitas 2005; Luz et al. 2006; Medeanic et al. 2006; Freitas et al. 2007; Toledo et al. 2009).

Based on palynological analysis of sediment from the Campos Basin, located in the northern region of the state of Rio de Janeiro, Brazil, Behling et al. (2002) observed changes in paleovegetation with a predominance of grasses in glacial cycles and increases in arboreal and semideciduous forests during interglacial cycles. Undertaking Pleistocene-Holocene palynological analysis of the BU91-GL-05 core, taken from the same basin, Freitas (2005) registered continental palynomorphs (cryptogamic spores, phanerogamic pollen grains, fresh water microalgae and microfungi). Vegetation types included taxa associated with Atlantic Forest (montane and lowland), semideciduous forest and restinga (coastal woodland), as well as herbs and hydrophytes. The percentage variations in the terrestrial palynomorphs indicated paleoenvironmental changes occurred during glacial and interglacial cycles over the past 145,000-150,000 years based on foraminiferal biozones. The morphological descriptions of spore and pollen types reveal a richness and diversity, in the same core, of pteridophyte and hydrophyte spores (Freitas et al. 2007) of periodically or totally flooded environments such as inundated brackish swamps, lagoon systems and flooded areas of pioneer formations of restinga, salt marshes and mangrove.

\footnotetext{
${ }^{1}$ Based on the Master's dissertation of the first author

${ }^{2}$ Universidade Federal do Rio de Janeiro, Museu Nacional, Departamento de Geologia e Paleontologia, Laboratório de Paleoecologia Vegetal, Rio de Janeiro, RJ, Brazil

${ }^{3}$ Universidade Federal do Rio de Janeiro, Museu Nacional, Departamento de Botânica, Laboratório de Palinologia, Rio de Janeiro, RJ, Brazil

${ }^{4}$ Author for correspondence: tuttyfreitas@gmail.com
} 
Palynological analyses coastal plain in northern part of the state of Rio de Janeiro indicate climatic oscillations from ca. $6,100{ }^{14} \mathrm{C}$ yr BP to the present. Luz \& Barth (2002) studied sediments from the nearby Lagoa de Cima, in the city of Campos dos Goytacazes, and registered spore-pollen indicators of Atlantic Forest, restinga, field vegetation, salt marshes and lagoon shores. The Holocene lacustrine sediment cores showed the expansion of the coastal plain with establishment of field vegetation between 6,500 and ca. $4,000{ }^{14} \mathrm{C}$ yr BP. These changes were probably mediated by relative sea level oscillations in the region during the Holocene (Luz et al. 2011).

In a paleoenvironmental reconstruction of the Lagoa Salgada, in the city of Macaé (state of Rio de Janeiro), Toledo et al. (2009) used multivariate statistical analyses in order to compare pollen records between surface and subsurface sediments. The authors determined that the recent vegetation became established in the region at ca. 2,500 ${ }^{14} \mathrm{C}$ yr BP.

As can be seen in Fig. 1, the current vegetation formations in the northern region of the state of Rio de Janeiro include the following (Radambrasil 1983; IBGE 1992): Atlantic Forest; semideciduous forest; and pioneer formations with fluvial and marine influence (restinga, mangrove and salt marshes).

This work presents morphological descriptions of pollen grains recovered from quaternary marine sediments (Fig. 1). These data were associated with recent vegetation in the northern region of the state of Rio de Janeiro and constitute the first records of terrestrial palynomorphs on the slope portion of the Campos Basin in southeastern Brazil.

\section{Study Area}

The Campos Basin is located in the southeastern portion of the Brazilian continental margin in an area between the states of Espírito Santo $\left(21^{\circ} \mathrm{S}\right.$, the Vitória ridge) and Rio de Janeiro $\left(23^{\circ} \mathrm{S}\right.$, the Cabo Frio upwelling) (Fig. 1). The studied region has a warm climate with seasonal humidity in lowland regions, with rainy summers and dry winters. The average monthly rainfall ranges from $650 \mathrm{~mm}$ to 1000 $\mathrm{mm}$, the dry season lasting 3-4 months (Nimer 1979). The average annual temperature in the largest city of the region (Campos dos Goytacazes) is $23^{\circ} \mathrm{C}$. The coastal plain, including the Paraiba do Sul river delta, is extensive. It gradually decreases between positions $21^{\circ} 30^{\prime} \mathrm{S}$ and $22^{\circ} 15^{\prime} \mathrm{S}$ and receives fluvial and marine influence (Nimer 1979; Radambrasil 1983).

The recent vegetation in the northern state of Rio de Janeiro comprises plant formations associated with Atlantic Forest (lowland and montane), semideciduous forest, mangrove and restinga (Radambrasil 1983; IBGE 1992). Phytosociological data for northern Rio de Janeiro (Assumpção \& Nascimento 2000; Costa \& Dias 2001; Moreno et al. 2003; Pessoa \& Oliveira 2006; Pimentel et al. 2007; Silva \& Nascimento 2001) suggest that the region encompasses the so-called tabuleiro forests (coastal lowland forests on Tertiary deposits) in the lowlands and xeromorphic (restinga) vegetation in the coastal areas.

In the north of the state of Rio de Janeiro, there is a major protected area (Jurubatiba Coastal Woodlands National Park), managed by the Chico Mendes Institute for

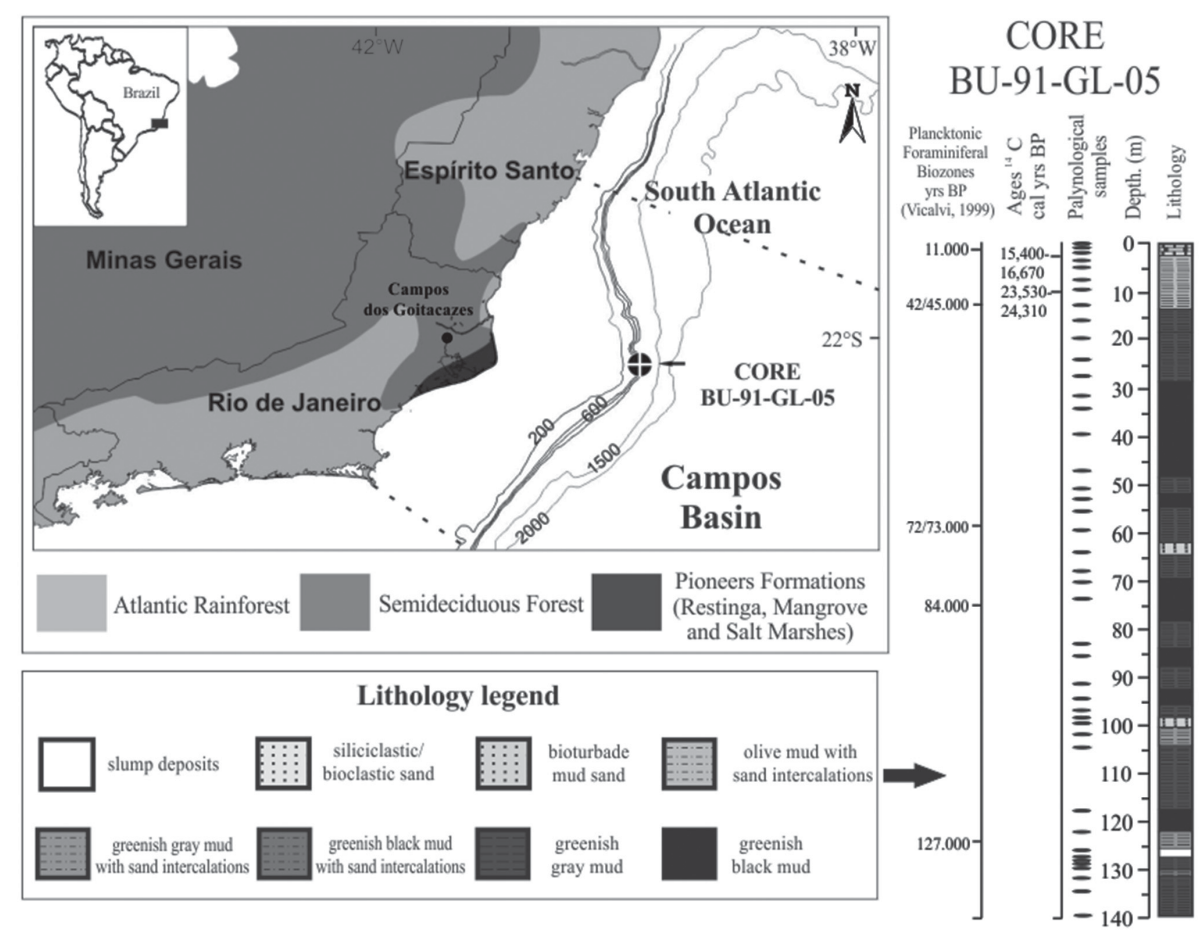

Figure 1. Map showing the location of core BU-91-GL-05, Albacora Slope, Campos Basin, state of Rio de Janeiro, Brazil, as well as the current distribution of vegetation in southeastern Brazil. 
the Conservation of Biodiversity, encompassing multiple restinga ecosystems (Costa \& Dias 2001; Pereira et al. 2004; Montezuma \& Araujo 2007).

\section{Material and methods}

The marine core BU-91-GL-05 (22 $08^{\prime} 12^{\prime \prime} \mathrm{S}$; $39^{\circ} 54^{\prime} 53^{\prime \prime} \mathrm{W}$ ) is $149.5 \mathrm{~m}$ long and was collected at a depth of $650 \mathrm{~m}$, on the Albacora Slope, northern Campos Basin, located $150 \mathrm{~km}$ east of the city of Campos dos Goytacazes, in the northern part of the state of Rio de Janeiro (Fig. 1). The core sediments are composed mainly of organic muds. Sandy intercalations occur near the top of the core, at $63-65 \mathrm{~m}$ and $97-100 \mathrm{~m}$. Slump or turbidite deposits also occur between 126.70$127.85 \mathrm{~m}$ (Caddah et al. 1998) (Fig. 1). Using planktonic foraminiferal biostratigraphy, Vicalvi $(1997 ; 1999)$ calculated the estimated age of deposition of the sediments in the core in the last 145,000-150,000 years. Two sediment samples from the top of the core were submitted to ${ }^{14} \mathrm{C}$-dating (Beta Analytic Inc., Miami, FL, USA). The ages derived were $13,210 \pm 70{ }^{14} \mathrm{C}$ yr BP for the 1.3-1.5 m interval and 20,020 $\pm 100{ }^{14} \mathrm{C}$ yr BP for the 10.2-10.4 m interval (Fig. 1; Tab. 1). Dates were calibrated using the IntCal09 curve (Ramsey et al. 2009) in the Calib 6.1.1 program (Stuiver \& Reimer 1993).

For pollen analysis, we selected 43 samples of core BU-91-GL-05 (10-20 g each). Samples were treated using the standard techniques employed in palynofacies analysis (Tyson 1995; Mendonça Filho et al. 2002), which consist of removing the mineral fraction, with hydrochloric and hydrofluoric acids, and concentrating the resulting organic residue with a dense liquid $\left(\mathrm{ZnCl}_{2} ; \mathrm{D}=2.0 \mathrm{~g} / \mathrm{cm}^{3}\right)$, in this case without oxidation. The samples were stored in the Laboratory of Plant Paleoecology at the National Museum of the Federal University of Rio de Janeiro.

We made standard morphological descriptions of pollen grains using characters such as polarity; symmetry; number, position and character of apertures; exine ornamentation; shape; and size (Punt et al. 2007) (Tab. 2). These characteristics provide a sound basis for correlations between fossil and modern pollen grains, permitting their taxonomic classification. The identification of pollen grains was made on the basis of data obtained from the following bibliographic sources: Moreira et al. (2004), Souza et al. (2004), Carrijo et al. (2005), Gonçalves-Esteves

Table 1. ${ }^{14} \mathrm{C}$ dating of core BU-91-GL-05, Albacora Slope, Campos Basin, state of Rio de Janeiro, Brazil.

\begin{tabular}{|c|c|c|c|c|}
\hline \multirow{2}{*}{ Lab code } & \multirow{2}{*}{ Depth (m) } & \multirow{2}{*}{${ }^{14} \mathrm{C}$ yrs BP } & Calibrated age & \multirow{2}{*}{$\delta^{13} \mathrm{C}$} \\
\hline & & & (cal yr BP) & \\
\hline Beta-270653 & 1.3 & $13,220 \pm 70$ & $15,400-16,670$ & $-25,4$ \\
\hline Beta-270652 & 10.2 & $20,020 \pm 100$ & $23,530-24,310$ & $-22,7$ \\
\hline
\end{tabular}

*Ages were calibrated based on Stuiver \& Reimer (1993) and Ramsey et al. (2009), with the IntCal09 calibration curve. et al. (2007), Mendonça et al. (2007a; 2007b; 2009; 2010), Aguiar et al. (2008), Peçanha et al. (2008), Loueuille et al. (2012) and Milward-Azevedo et al. (2010; 2012). This was in addition to consulting the reference collection of the Álvaro Xavier Moreira Laboratory of Palynology in the Botany Department of the National Museum of the Federal University of Rio de Janeiro. The pollen type was characterized by establishing the proximity between the material analyzed and the taxonomic group (Salgado-Labouriau 1973; Lorscheitter 1989). The photomicrographs shown in Fig. 2 and Fig. 3 were obtained with a Zeiss light microscope in conjunction with the AxioVision software, version 6.0 (Carl Zeiss, Oberkochen, Germany).

Pollen descriptions (Tab. 2) follow the classification system established in the Angiosperm Phylogeny Group III guidelines (Souza \& Lorenzi 2008; , APG III 2009). The pollen types identified in core BU-91-GL-05 correspond to recent vegetation reported for Atlantic Forest (lowland and montane), semideciduous forest and restinga, as well as to the hygrophytes, herbs and other vegetation currently occurring in flooded coastal plain environments. These correlations were based on floristic and phytosociological surveys carried out on the northern of the Rio de Janeiro State (Tab. 3).

\section{Results and discussion}

In the 43 samples taken from core BU-91-GL-05, we identified 46 pollen types (Fig. 2 and 3). These were distributed as follows (Tab. 2 and 3): one gymnosperm genus (Podocarpus); and 45 angiosperm types belonging to 27 families-15 types of a single family of monocotyledons (Poaceae) and 30 types across 26 families of dicotyledons. The families of angiosperms that were most representative in terms of the number of pollen types were Fabaceae, with four (Fabaceae type, Bauhinia, Inga and Canavalia), Amaranthaceae, with three (Chenopodium, Amaranthus and Gomphrena), Malpighiaceae, with three (Tetrapteris, Heteropteris and Peixotoa), Malvaceae, with three (Sida, Abutilon, Hibiscus and Pseudobombax), Rubiaceae, with three (Faramea, Borreria and Psychotria), Bignoniaceae, with three (Bignoniaceae type, Adenocalymma and Tabebuia), Asteraceae, with two (Eupatorium and tribe Vernonieae), and Onagraceae, with two (Fuchsia and Ludwigia). The wide spectrum of pollen types points to a dynamic paleovegetation environment throughout the Upper Pleistocene-Holocene in southeastern Brazil, especially in the north of the state of Rio de Janeiro.

\section{Relationships between fossil pollen records and recent vegetation}

The pollen types identified are associated with Atlantic Forest (lowland and montane), semideciduous forest and pioneer formations (restinga, mangrove and salt marshes) 
Table 2. Morphological descriptions of pollen grains recorded from core BU-91-GL-05, Albacora Slope, Campos Basin, state of Rio de Janeiro, Brazil.

\begin{tabular}{|c|c|c|c|c|c|c|c|}
\hline \multicolumn{8}{|l|}{$\begin{array}{l}\text { Pollen grain distribution } \\
\text { CLASS-SUBCLASS }\end{array}$} \\
\hline Family & $\begin{array}{l}\text { Pollen } \\
\text { unit }\end{array}$ & $\begin{array}{l}\text { Polarity/ } \\
\text { Symmetry }\end{array}$ & Size $(\mu \mathrm{m})$ & NPC apertures & Exine & Shape & Observations \\
\hline \multicolumn{8}{|l|}{ GYMNOSPERMAE } \\
\hline \multicolumn{8}{|l|}{ Podocarpaceae } \\
\hline $\begin{array}{l}\text { Type } 1 \text { Podocarpus L’Her. ex Pers. } \\
\text { [Fig. 2; 1] }\end{array}$ & monad & $\begin{array}{l}\text { apolar- } \\
\text { asymmetric }\end{array}$ & $62.0 \times 33.0$ & bisaccate & reticulate & & \\
\hline \multicolumn{8}{|l|}{ ANGIOSPERMAE-MONOCOTYLEDONES } \\
\hline \multicolumn{8}{|l|}{ Poaceae } \\
\hline Type 2 Poaceae [Fig. 2; 2] & monad & $\begin{array}{l}\text { heteropolar- } \\
\text { radiosymmetric }\end{array}$ & $42.0 \times 38.0$ & monoporate & psilate to scabrate & spheroidal & $\begin{array}{l}\text { pore with } \\
\text { conspicuous } \\
\text { annulus }\end{array}$ \\
\hline \multicolumn{8}{|l|}{ ANGIOSPERMAE-DICOTYLEDONES } \\
\hline \multicolumn{8}{|l|}{ Acanthaceae } \\
\hline Type 3 Acanthaceae [Fig. 2; 3] & monad & $\begin{array}{l}\text { isopolar- } \\
\text { radiosymmetric }\end{array}$ & $45.8 \times 34.0$ & 3-zonoporate & $\begin{array}{l}\text { reticulate- } \\
\text { baculate }\end{array}$ & prolate & \\
\hline \multicolumn{8}{|l|}{ Amaranthaceae } \\
\hline Type 4 Chenopodium L. [Fig. 2; 4] & monad & $\begin{array}{l}\text { apolar- } \\
\text { asymmetric }\end{array}$ & $20.0 \times 20.0$ & $\begin{array}{l}\text { pantoporate } \\
\text { ( } 42 \text { pores) }\end{array}$ & microreticulate & spheroidal & \\
\hline Type 5 Amaranthus L. [Fig. 2; 5] & monad & $\begin{array}{l}\text { apolar- } \\
\text { asymmetric }\end{array}$ & $26.0 \times 26.0$ & $\begin{array}{l}\text { pantoporate } \\
\text { (7 pores) }\end{array}$ & granulate & spheroidal & \\
\hline Type 6 Gomphrena [Fig. 2; 6-7] & monad & $\begin{array}{l}\text { apolar- } \\
\text { asymmetric }\end{array}$ & $\begin{array}{c}44.0 \times \\
44.0 ; 33.3 \\
\times 33.3\end{array}$ & $\begin{array}{l}\text { pantoporate } \\
\text { (12 pores) }\end{array}$ & lophate & spheroidal & \\
\hline \multicolumn{8}{|l|}{ Apocynaceae } \\
\hline Type 7 Apocynaceae [Fig. 2; 8] & monad & $\begin{array}{l}\text { isopolar- } \\
\text { radiosymmetric }\end{array}$ & $52.0 \times 50.0$ & 3-zonoporate & $\begin{array}{l}\text { scabrate to } \\
\text { rugulate }\end{array}$ & $\begin{array}{c}\text { oblate } \\
\text { spheroidal }\end{array}$ & \\
\hline \multicolumn{8}{|l|}{ Aquifoliaceae } \\
\hline Type 8 Ilex L. [Fig. 2;9] & monad & $\begin{array}{l}\text { isopolar- } \\
\text { radiosymmetric }\end{array}$ & $32.2 \times 28.6$ & tricolporate & clavate & $\begin{array}{l}\text { prolate } \\
\text { spheroidal }\end{array}$ & \\
\hline \multicolumn{8}{|l|}{ Asteraceae } \\
\hline Type 9 Eupatorium [Fig. 2; 10] & monad & $\begin{array}{l}\text { isopolar- } \\
\text { radiosymmetric }\end{array}$ & $32.0 \times 30.0$ & tricolporate & echinate & $\begin{array}{l}\text { oblate } \\
\text { spheroidal }\end{array}$ & \\
\hline $\begin{array}{l}\text { Type } 10 \text { Tribe Vernonieae } \\
\text { [Fig. } 2 ; 11 \text { ] }\end{array}$ & monad & $\begin{array}{l}\text { isopolar- } \\
\text { radiosymmetric }\end{array}$ & $50.0 \times 47.0$ & tricolporate & subechinolophate & $\begin{array}{l}\text { oblate } \\
\text { spheroidal } \\
\text { to prolate }\end{array}$ & \\
\hline \multicolumn{8}{|l|}{ Bignoniaceae } \\
\hline Type 11 Bignoniaceae [Fig. 2; 13] & monad & $\begin{array}{l}\text { isopolar- } \\
\text { radiosymmetric }\end{array}$ & $40.0 \times 27.2$ & tricolporate & $\begin{array}{c}\text { reticulate- } \\
\text { homobrochate }\end{array}$ & prolate & \\
\hline $\begin{array}{l}\text { Type } 12 \text { Adenocalymma } \\
\text { Mart.ex Meisn. [Fig. 2; 14] }\end{array}$ & monad & $\begin{array}{l}\text { apolar- } \\
\text { asymmetric }\end{array}$ & $45.0 \times 42.0$ & inaperturate & rugulate & spheroidal & \\
\hline $\begin{array}{l}\text { Type } 13 \text { Tabebuia Gomes } \\
\text { ex. DC. [Fig. } 2 ; 15 \text { ] }\end{array}$ & monad & $\begin{array}{l}\text { isopolar- } \\
\text { radiosymmetric }\end{array}$ & $36.6 \times 34.8$ & tricolporate & $\begin{array}{l}\text { reticulate- } \\
\text { homobrochate }\end{array}$ & prolate & \\
\hline \multicolumn{8}{|l|}{ Boraginaceae } \\
\hline Type 14 Tournefortia L. [Fig. 2; 16] & monad & isopolar & $20.0 \times 18.0$ & tricolporate & $\begin{array}{l}\text { gemmate- } \\
\text { verrucate }\end{array}$ & & \\
\hline \multicolumn{8}{|l|}{ Clusiaceae } \\
\hline Type 15 Garcinia L. [Fig. 2; 18] & monad & $\begin{array}{l}\text { isopolar- } \\
\text { radiosymmetric }\end{array}$ & $41.4 \times 14.8$ & tricolporate & rugulate & $\begin{array}{l}\text { prolate } \\
\text { spheroidal }\end{array}$ & \\
\hline \multicolumn{8}{|l|}{ Convolvulaceae } \\
\hline Type 16 Merremia [Fig. 2; 17] & monad & $\begin{array}{l}\text { isopolar- } \\
\text { radiosymmetric }\end{array}$ & $60.0 \times 58.4$ & tricolpate & $\begin{array}{l}\text { reticulate with } \\
\text { granula }\end{array}$ & $\begin{array}{c}\text { oblate } \\
\text { spheroidal }\end{array}$ & \\
\hline \multicolumn{8}{|l|}{ Ericaceae } \\
\hline Type 17 Ericaceae [Fig. 2; 19] & tetrad & $\begin{array}{l}\text { isopolar- } \\
\text { radiosymmetric }\end{array}$ & $29.6 \times 29.6$ & $\begin{array}{l}\text { tricolporate } \\
\text { demicolpate }\end{array}$ & reticulate & & \\
\hline \multicolumn{8}{|l|}{ Euphorbiaceae } \\
\hline Type 18 Sebastiania [Fig. 2; 20] & monad & $\begin{array}{l}\text { isopolar- } \\
\text { radiosymmetric }\end{array}$ & & tricolporate & microreticulate & $\begin{array}{c}\text { prolate } \\
\text { spheroidal }\end{array}$ & \\
\hline Type 19 Alchornea Sw. [Fig. 2;21] & monad & $\begin{array}{l}\text { isopolar- } \\
\text { radiosymmetric }\end{array}$ & $28.0 \times 27.7$ & tricolporate & reticulate & $\begin{array}{l}\text { suboblate } \\
\text { to oblate } \\
\text { spheroidal }\end{array}$ & \\
\hline Type 20 Croton L. [Fig. 2; 22] & monad & apolar & $40.0 \times 40.0$ & inaperturate & croton pattern & spheroidal & \\
\hline
\end{tabular}


Table 2. Continuation

\begin{tabular}{|c|c|c|c|c|c|c|c|}
\hline \multicolumn{8}{|l|}{ Pollen grain distribution } \\
\hline \multicolumn{8}{|l|}{ CLASS-SUBCLASS } \\
\hline $\begin{array}{c}\text { Family } \\
\text { Type }\end{array}$ & $\begin{array}{l}\text { Pollen } \\
\text { unit }\end{array}$ & $\begin{array}{l}\text { Polarity/ } \\
\text { Symmetry }\end{array}$ & Size $(\mu \mathrm{m})$ & NPC apertures & Exine & Shape & Observations \\
\hline \multicolumn{8}{|l|}{ Fabaceae } \\
\hline Type 21 Fabaceae [Fig. 2; 23] & monad & $\begin{array}{l}\text { isopolar- } \\
\text { radiosymmetric }\end{array}$ & $37.0 \times 34.1$ & tricolpate & scabrate & spheroidal & $\begin{array}{l}\text { equatorial } \\
\text { view }\end{array}$ \\
\hline Type 22 Bauhinia L. [Fig. 2; 24] & monad & $\begin{array}{c}\text { isopolar- } \\
\text { radiosymmetric }\end{array}$ & $34.7 \times 33.0$ & tricolporate & $\begin{array}{l}\text { reticulate with } \\
\text { supratectal } \\
\text { gemmae }\end{array}$ & subprolate & \\
\hline Type 23 Inga Scop. [Fig. 2; 25] & polyad & $\begin{array}{c}\text { apolar- } \\
\text { asymmetric }\end{array}$ & $60.0 \times 57.0$ & triporate & psilate & & $\begin{array}{l}16 \text { pollen } \\
\text { grains }\end{array}$ \\
\hline Type 24 Canavalia DC. [Fig. 2; 26] & monad & $\begin{array}{c}\text { isopolar- } \\
\text { radiosymmetric }\end{array}$ & $46.0 \times 42.0$ & tricolporate & $\begin{array}{l}\text { rugulate- } \\
\text { perforate }\end{array}$ & suboblate & \\
\hline \multicolumn{8}{|l|}{ Lentibulariaceae } \\
\hline Type 25 Utricularia L. [Fig. 2; 27] & monad & $\begin{array}{c}\text { isopolar- } \\
\text { radiosymmetric }\end{array}$ & $53.0 \times 43.5$ & 14-17-colporate & psilate & $\begin{array}{l}\text { oblate } \\
\text { spheroidal } \\
\text { to suboblate }\end{array}$ & \\
\hline \multicolumn{8}{|l|}{ Loranthaceae } \\
\hline Type 26 Struthanthus Mart. [Fig. 3; 1] & monad & $\begin{array}{c}\text { isopolar- } \\
\text { radiosymmetric }\end{array}$ & $27.5 \times 27.2$ & syncolporate & scabrate & $\begin{array}{l}\text { peroblate to } \\
\text { suboblate }\end{array}$ & \\
\hline \multicolumn{8}{|l|}{ Malpighiaceae } \\
\hline Type 27 Tetrapteris Cav. [Fig. 3;2] & monad & $\begin{array}{l}\text { apolar- } \\
\text { asymmetric }\end{array}$ & $\begin{array}{c}27.0-35.0 \times \\
23.0-33.0\end{array}$ & porate ( 6 pores) & $\begin{array}{l}\text { rugulate to } \\
\text { microreticulate }\end{array}$ & spheroidal & \\
\hline Type 28 Heteropteris Fee. [Fig. 3; 3] & monad & $\begin{array}{l}\text { apolar- } \\
\text { radiosymmetric }\end{array}$ & $\begin{array}{c}27.0-31.0 \times \\
27.0-28.0\end{array}$ & $\begin{array}{l}\text { zonoporate (6-8 } \\
\text { pores) }\end{array}$ & $\begin{array}{l}\text { scabrate/ } \\
\text { rugulate/ } \\
\text { perforate }\end{array}$ & spheroidal & \\
\hline Type 29 Peixotoa Juss. [Fig. 3; 4] & monad & $\begin{array}{c}\text { apolar- } \\
\text { radiosymmetric }\end{array}$ & $27.0 \times 26.0$ & $\begin{array}{l}\text { zonoporate (8 } \\
\text { pores) }\end{array}$ & perforate & spheroidal & \\
\hline \multicolumn{8}{|l|}{ Malvaceae } \\
\hline Type 30 Sida [Fig. 3; 6] & monad & $\begin{array}{l}\text { apolar- } \\
\text { asymmetric }\end{array}$ & $\begin{array}{c}42.8-48.5 \times \\
40.9-44.2\end{array}$ & $\begin{array}{l}\text { pantoporate (30 } \\
\text { pores) }\end{array}$ & echinate & spheroidal & \\
\hline Type 31 Abutilon Mill. [Fig. 3; 5] & monad & $\begin{array}{l}\text { apolar- } \\
\text { asymmetric }\end{array}$ & $\begin{array}{c}42.7-56.6 \times \\
42.7-57.8\end{array}$ & tricolporate & echinate & spheroidal & \\
\hline Type 32 Hibiscus L. [Fig. 3; 7] & monad & $\begin{array}{c}\text { apolar- } \\
\text { asymmetric }\end{array}$ & $\begin{array}{c}50.0-69.3 \times \\
50.0-73.3\end{array}$ & pantoporate & echinate & spheroidal & \\
\hline Type 33 Pseudobombax Dugand [Fig. 3;8] & monad & $\begin{array}{c}\text { isopolar- } \\
\text { radiosymmetric }\end{array}$ & $60.0 \times 55.5$ & brevitricolporate & $\begin{array}{c}\text { reticulate- } \\
\text { heterobrochate }\end{array}$ & oblate & \\
\hline \multicolumn{8}{|l|}{ Meliaceae } \\
\hline Type 34 Meliaceae [Fig. 3; 9] & monad & $\begin{array}{c}\text { isopolar- } \\
\text { radiosymmetric }\end{array}$ & $34.5 \times 34.5$ & $\begin{array}{c}\text { zonocolporate } \\
\text { (4-colporate) }\end{array}$ & reticulate & spheroidal & \\
\hline \multicolumn{8}{|l|}{ Myrtaceae } \\
\hline Type 35 Myrtaceae [Fig. 3; 10] & monad & $\begin{array}{c}\text { isopolar- } \\
\text { radiosymmetric }\end{array}$ & $21.8-23.0$ & tricolporate & psilate & $\begin{array}{l}\text { peroblate } \\
\text { to oblate } \\
\text { spheroidal }\end{array}$ & \\
\hline \multicolumn{8}{|l|}{ Onagraceae } \\
\hline Type 36 Fuchsia L. [Fig. 3; 11] & monad & $\begin{array}{l}\text { heteropolar- } \\
\text { radiosymmetric }\end{array}$ & $\begin{array}{c}60.0-70.0 \times \\
50.0-68.0\end{array}$ & triporate & psilate to scabrate & $\begin{array}{l}\text { suboblate to } \\
\text { oblate }\end{array}$ & \\
\hline Type 37 Ludwigia L. [Fig. 3; 12] & monad & $\begin{array}{l}\text { heteropolar- } \\
\text { radiosymmetric }\end{array}$ & $\begin{array}{c}44.7-56.6 \times \\
44.4-59.0\end{array}$ & tricolporate & rugulate & $\begin{array}{l}\text { suboblate to } \\
\text { oblate }\end{array}$ & \\
\hline \multicolumn{8}{|l|}{ Passifloraceae } \\
\hline Type 38 Passiflora L. [Fig. 3; 13] & monad & $\begin{array}{c}\text { isopolar- } \\
\text { radiosymmetric }\end{array}$ & $\begin{array}{c}43.2-45.7 \times \\
32.8-37.1\end{array}$ & zonocolpate & $\begin{array}{c}\text { reticulate- } \\
\text { homobrochate }\end{array}$ & $\begin{array}{l}\text { oblate to } \\
\text { subprolate }\end{array}$ & \\
\hline \multicolumn{8}{|l|}{ Polygalaceae } \\
\hline Type 39 Polygala L. [Fig. 3; 15] & monad & $\begin{array}{c}\text { isopolar- } \\
\text { radiosymmetric }\end{array}$ & $37.8 \times 32.8$ & $\begin{array}{l}\text { zonocolporate } \\
\text { (8-10 colpori) }\end{array}$ & rugulate & subprolate & \\
\hline \multicolumn{8}{|l|}{ Polygonaceae } \\
\hline Type 40 Polygonum L. [Fig. 3; 14] & monad & apolar & $\begin{array}{c}39.0-47.0 \times \\
35.2-40.0\end{array}$ & pantoporate & $\begin{array}{c}\text { reticulate- } \\
\text { heterobrochate }\end{array}$ & spheroidal & \\
\hline
\end{tabular}


Table 2. Continuation.

\begin{tabular}{|c|c|c|c|c|c|c|c|}
\hline Pollen grain distribution & & & & & & & \\
\hline CLASS-SUBCLASS & & & & & & & \\
\hline $\begin{array}{l}\text { Family } \\
\text { Type }\end{array}$ & $\begin{array}{l}\text { Pollen } \\
\text { unit }\end{array}$ & $\begin{array}{l}\text { Polarity/ } \\
\text { Symmetry }\end{array}$ & Size $(\mu \mathrm{m})$ & NPC apertures & Exine & Shape & Observations \\
\hline Rubiaceae & & & & & & & \\
\hline Type 41 Faramea [Fig. 3; 16] & monad & $\begin{array}{c}\text { isopolar- } \\
\text { radiosymmetric }\end{array}$ & $34.0 \times 30.0$ & triporate & rugulate & $\begin{array}{c}\text { oblate- } \\
\text { spheroidal }\end{array}$ & \\
\hline Type 42 Borreria Mey. [Fig. 3; 17] & monad & $\begin{array}{l}\text { isopolar- } \\
\text { radiosymmetric }\end{array}$ & $44.0 \times 40.0$ & $\begin{array}{l}\text { zonocolporate } \\
\text { (10-colporate) }\end{array}$ & spiculate-baculate & suboblate & \\
\hline Type 43 Psychotria L. [Fig. 3; 18] & monad & apolar & $37.0 \times 36.0$ & inaperturate & reticulate & $\begin{array}{l}\text { spheroidal } \\
\text { to } \\
\text { subprolate }\end{array}$ & \\
\hline Sapindaceae & & & & & & & \\
\hline Type 44 Sapindaceae [Fig. 3; 19] & monad & $\begin{array}{c}\text { isopolar- } \\
\text { radiosymmetric }\end{array}$ & $58.8 \times 34.4$ & triporate & scabrate & $\begin{array}{l}\text { suboblate to } \\
\text { peroblate }\end{array}$ & \\
\hline Solanaceae & & & & & & & \\
\hline Type 45 Solanaceae [Fig. 3; 20] & monad & $\begin{array}{l}\text { isopolar- } \\
\text { radiosymmetric }\end{array}$ & $27.6 \times 28.0$ & tricolporate & rugulate & $\begin{array}{l}\text { prolate- } \\
\text { spheroidal }\end{array}$ & \\
\hline Symplocaceae & & & & & & & \\
\hline Type 46 Symplocos [Fig. 2; 12] & monad & $\begin{array}{c}\text { isopolar- } \\
\text { radiosymmetric }\end{array}$ & $32.5 \times 32.5$ & triporate & scabrate & circular & \\
\hline
\end{tabular}

NPC - number, position and character.

Table 3. Pollen types registered in core BU-91-GL-05 and their associated ecosystems. According with: Garcia (1994); Rizzini (1997); Assumpção \& Nascimento (2000); Kurtz \& Araujo (2000); Pereira \& Araujo (2000); Costa \& Dias (2001); Silva \& Nascimento (2001); Bove et al. (2003); Moreno et al. (2003); Pessoa \& Oliveira (2006); Pimentel et al. (2007); Souza \& Lorenzi (2008).

\begin{tabular}{|c|c|c|c|}
\hline Ecosystems & Family - Pollen type & Ecosystems & Family - Pollen type \\
\hline \multirow{3}{*}{ Montane forest } & Podocarpaceae - Podocarpus & Restinga & Ericaceae - Ericaceae Type \\
\hline & Symplocaceae - Symplocos & & Fabaceae - Canavalia \\
\hline & Onagraceae - Fuchsia & & Malvaceae - Abutilon \\
\hline Lowland formations & & & Malvaceae - cf. Hibiscus \\
\hline \multirow{7}{*}{$\begin{array}{l}\text { Atlantic Forest and } \\
\text { semideciduous forest }\end{array}$} & Acanthaceae - Acanthaceae Type & & Polygalaceae - Polygala \\
\hline & Apocynaceae - Apocynaceae Type & & \\
\hline & Bignoniaceae - Bignoniaceae Type & Aquatics & Lentibulariaceae - Utricularia \\
\hline & Meliaceae- Meliaceae Type & & Onagraceae - Ludwigia \\
\hline & Sapindaceae- Sapindaceae Type & & Polygonaceae Polygonum \\
\hline & Solanaceae - Solanaceae Type & & \\
\hline & & Herbs & Asteraceae - Eupatorieae \\
\hline \multirow{14}{*}{$\begin{array}{l}\text { Atlantic Forest and } \\
\text { semideciduous forest/Restinga }\end{array}$} & Aquifoliaceae - Ilex & & Asteraceae - Vernonieae \\
\hline & Clusiaceae- Garcinia & & Poaceae - Poaceae Type \\
\hline & Euphorbiaceae - Alchornea & & Rubiaceae - Borreria \\
\hline & Euphorbiaceae - Euphorbiaceae Type & & Rubiaceae - Faramea \\
\hline & Fabaceae - Inga & & \\
\hline & Fabaceae - Fabaceae Type & Flooded coastal plain environments & Poaceae - Poaceae Type \\
\hline & Malpighiaceae - Tetrapteris & & Aquifoliaceae - Ilex \\
\hline & Malpighiaceae - Heteropteris & & Asteraceae - Eupatorium \\
\hline & Malvaceae - Pseudobombax & & Clusiaceae - Garcinia \\
\hline & Malvaceae - Sida & & Euphorbiaceae - Alchornea \\
\hline & Myrtaceae - Myrtaceae Type & & Fabaceae - Inga \\
\hline & Passifloraceae - Passiflora & & Lentibulariaceae - Utricularia \\
\hline & Rubiaceae- Psychotria & & Malvaceae - Hibiscus \\
\hline & & & Onagraceae - Ludwigia \\
\hline \multirow[t]{5}{*}{ Restinga } & Amaranthaceae - Amaranthus & & Passifloraceae - Passiflora \\
\hline & Amaranthaceae - Chenopodium & & Polygalaceae - Polygala \\
\hline & Amaranthaceae - Gomphrena & & Polygonaceae - Polygonum \\
\hline & Boraginaceae Tournefortia & & Rubiaceae - Borreria \\
\hline & Convolvulaceae Convolvulaceae Type & & \\
\hline
\end{tabular}

${ }^{*}$ Montane and lowland formations comprise the Atlantic Forest. 
distributed at different elevations in the north of the state of Rio de Janeiro (Radambrasil 1983). To make those associations, we consulted floristic studies undertaken in montane and submontane areas (Kurtz \& Araujo 2000; Moreno et al. 2003; Pessoa \& Oliveira 2006); dense lowland forest and semideciduous forest (Silva \& Nascimento 2001; Kurtz et al. 2009); restinga (Costa \& Dias 2001; Pimentel et al. 2007); and herbaceous and hygrophytic coastal vegetation ecosystems (IBGE 1992; Costa \& Dias 2001; Bove et al. 2003).

\section{Atlantic Forest}

Arboreal taxa (Podocarpus and Ilex), as classified by the Brazilian Institute of Geography and Statistics (IBGE 1992) and Radambrasil (1983), are tree layer components derived from mixed and montane/upper montane Araucaria forest, at 400-2,000 m of elevation. Arboreal taxa of lowland tropical rain forest do occur, as represented by Euphorbiaceae (Alchornea and Croton), Myrtaceae and Rubiaceae, which occupy the slopes of coastal and inland mountains (Radambrasil 1983; IBGE 1992). According to Carauta \& Rocha (1988), the Podocarpaceae arboreal family consists of high latitude woodlands along the length of the Paraíba do Sul River, in the state of Rio de Janeiro.

Moreno et al. (2003) undertook a phytosociological survey in two areas of dense, submontane forest, at 50-250 m of elevation, in the foothills of the Imbé mountain range, near Campos dos Goytacazes, in the state of Rio de Janeiro (Fig. 1). The families Fabaceae, Myrtaceae, Euphorbiaceae and Rubiaceae were found to be quite species-rich in the area. The taxa most commonly found in the marine sediment examined in the present study (Tabebuia, Alchornea, Inga, Meliaceae, Trichilia, Myrtaceae, Psychotria and Symplocos) were also recorded for the Imbé region, on the coastal plain of the northern part of the state of Rio de Janeiro. Phytosociological studies of the shrub-arboreal component of a submontane forest in the Poço das Antas Biological Preserve (state of Rio de Janeiro) have shown that the most common botanical families are Euphorbiaceae, Fabaceae, Myrtaceae, Bignoniaceae, Sapindaceae, Boraginaceae, Meliaceae and Rubiaceae (Pessoa \& Oliveira 2006). Our pollen data fit with the Garcinia, Inga, Myrtaceae, Alchornea, Asteraceae and Psychotria taxa. Some of the arboreal taxa recorded for the Atlantic Forest in the Paradise State Ecological Reserve, near the town of Cachoeira de Macacu, in the state of Rio de Janeiro (Tabebuia, Garcinia, Meliaceae, Myrtaceae and Psychotria) (Kurtz \& Araujo 2000) were also seen in marine sediments of the Campos Basin.

The shrub-herbaceous component of the lowland Atlantic Forest is composed of Adenocalymma, Arrabidaea, Cordia, Vernonia, Alchornea, Poaceae, Desmodium, Inga, Hibiscus, Guarea, Myrtaceae, Polygonum and Psychotria (Vieira \& Pessoa, 2001). Occurring on flood plains, hills and coastal plains, the pollen types of these taxa are similar to those recorded in core BU-91-GL-05, characteristic of dense submontane forest vegetation.

\section{Semideciduous forest}

In floristic surveys of the Mata do Carvão forest, a remnant of the Mata Tabuleiros forest, located near Campos dos Goytacazes, Silva \& Nascimento (2001) noted the species richness of the arboreal families Fabaceae, Euphorbiaceae, Bignoniaceae, Meliaceae, Myrtaceae, Sapindaceae and Rubiaceae. In sediment from the Albacora Slope, we found Tabebuia, Pseudobombax, Myrtaceae, Meliaceae, Polygala and Rubiaceae. Phytosociological data from the Pau Brasil Environmentally Protected Area, in the Emerenças Hills, near the city of Armação dos Búzios, which is in the lakes region of the state of Rio de Janeiro, indicate the dominance of Myrtaceae, Fabaceae and Euphorbiaceae (Kurtz et al. 2009).

Some of the taxa recorded in the present study (Tabebuia, Garcinia, Heteropteris, Pseudobombax, Meliaceae and Myrtaceae) belong to the shrub-arboreal component of semideciduous forests in coastal regions of the state of Rio de Janeiro. Bauhinia (Fabaceae) was also recorded by Kurtz et al. (2009) and is present in the lowland forest arboreal layer occupying the hills and coastal plains (Costa \& Dias 2001).

\section{Restinga}

Considering the diversity of pollen types identified and registered, as well as the proximity to the restingas in the study area, those pollen types have strong relationships with arboreal taxa of shrub and herbaceous vegetation, restinga and other pioneer formations. According to Rizzini (1997), restingas are found in regions with direct marine influence and consequently exhibit an enormous variety of vegetation types. Coastal area vegetation communities are directly affected by tidal fluctuations. The families (genera) commonly found near beaches and dunes include the following (IBGE 1992; Radambrasil 1983): Fabaceae (Canavalia), Poaceae (Paspalum), Apiaceae (Hydrocotyle), Erythroxylaceae (Erythroxylum), and Myrtaceae.

In a floristic analysis of restingas in the state of Rio de Janeiro, Pereira \& Araujo (2000) showed that the most representative families are Fabaceae, Myrtaceae, Orchidaceae, Bromeliaceae, Rubiaceae, Cyperaceae, Asteraceae, Poaceae, Melastomataceae and Euphorbiaceae. The taxa identified in our study have also been reported in floristic and phytosociological studies of the restingas of Rio de Janeiro, especially along its northern coast. Among the plant communities in Jurubatiba Coastal Woodlands National Park, the following related herbaceous types have been recorded (Costa \& Dias 2001): Amaranthaceae (Alternanthera, Blutaparon and Gomphrena); Apocynaceae; Ilex; Asteraceae (Eupatorium and Vernonia); Bignoniaceae (Adenocalymma, Arrabidaea and Tabebuia); Malvaceae (Pseudobombax, Abutilon and $\mathrm{Hi}$ biscus); Boraginaceae (Tournefortia); Clusiaceae (Garcinia); Ericaceae; Euphorbiaceae (Alchornea and Croton); Fabaceae (Bauhinia, Canavalia and Inga); Lentibulariaceae (Utricula- 

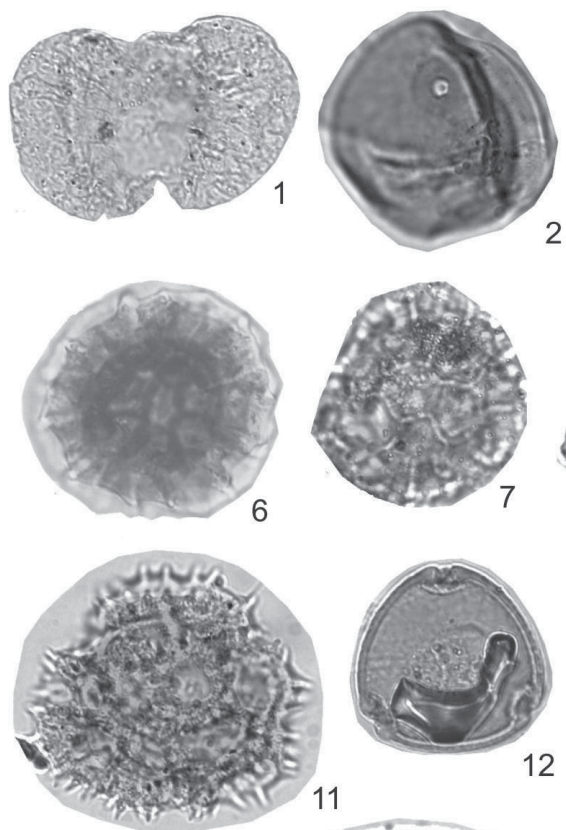

12
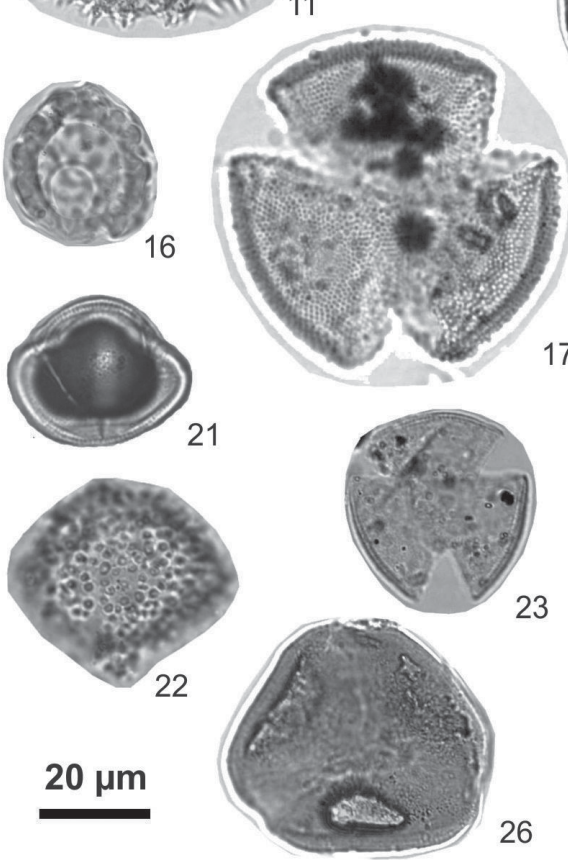
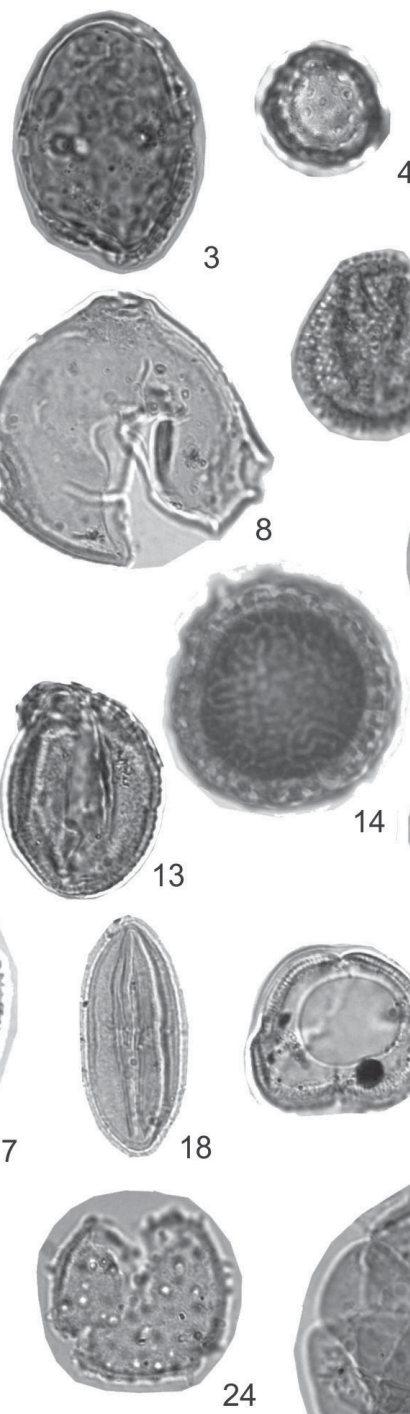

4
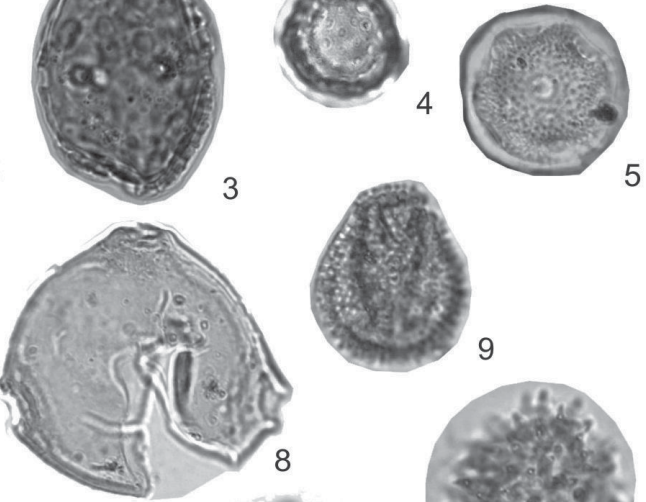

9
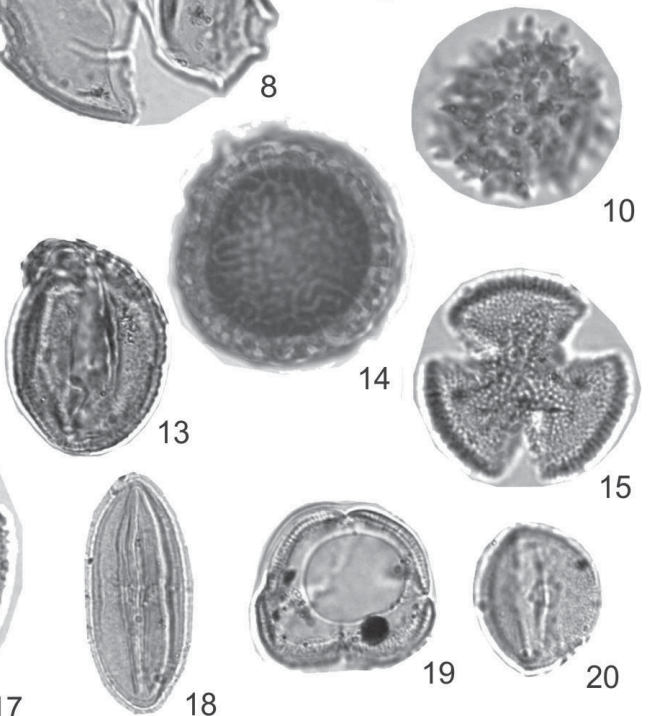

15

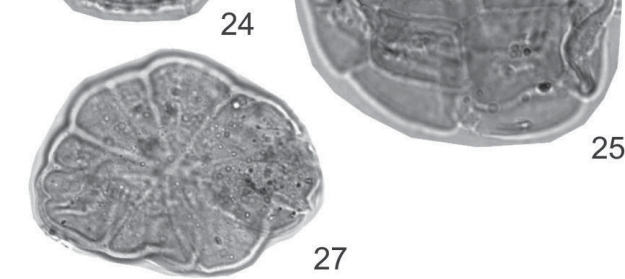

Figure 2. Photomicrographs of gymnosperm and angiosperm pollen grains registered in core BU-91-GL-05 (magnification, $\geq 1000 \times$ ): 1 -Type 1 Podocarpus; 2-Type 2 Poaceae; 3-Type 3 Acanthaceae; 4 -Type 4 Chenopodium; 5-Type 5 Amaranthus; 6,7-Type 6 Gomphrena; 8-Type 7 Apocynaceae; 9-Type 8 Ilex; 10-Type 9 Eupatorium; 11-Type 10 Vernonia; 12-Type 11 Symplocos; 13-Type 12 Bignoniaceae; 14 -Type 13 Adenocalymma; 15-Type 14 Tabebuia; 16-Type 15 Tournefortia; 17-Type 16 Merremia; 18-Type 17 Garcinia; 19-Type 18 Ericaceae; 20-Type 19 Sebastiania; 21-Type 20 Alchornea; 22-Type 21 Croton; 23-Type 22 Fabaceae; 24-Type 23 Bauhinia; 25-Type 24 Inga; 26-Type 25 Canavalia; 27-Type 26 Utricularia. Scale: $20 \mu \mathrm{m}$.

ria); Malpighiaceae (Heteropteris, Tetrapteris and Peixotoa); Meliaceae; Myrtaceae; Onagraceae (Ludwigia); Passifloraceae (Passiflora); Poaceae; Polygala (Polygala); Rubiaceae (Faramea, Borreria and Psychotria); and Sapindaceae. According to Araujo (2000), restinga is compartmentalized into various vegetation formations. The taxa identified in the Campos Basin marine sediments were similar to those occurring in such formations: herbaceous formations on sandbanks (Poaceae, Blutaparon, Alternanthera, Gomphre$n a$ and Canavalia); dense shrubby backshore formations (Poaceae, Vernonia and Erythroxylum); sparse shrubby Clusia-dominated formations (Poaceae, Lepidaploa, Vernonia, Peixotoa, Croton, Faramea, Ilex and Paullinia); sparse shrubby Ericaceae-dominated formations (Ericaceae, Poaceae, Vernonia, Peixotoa, Croton and Ilex); restingas sensu stricto (Alternanthera, Myrtaceae, Garcinia, Alchornea, Croton, 


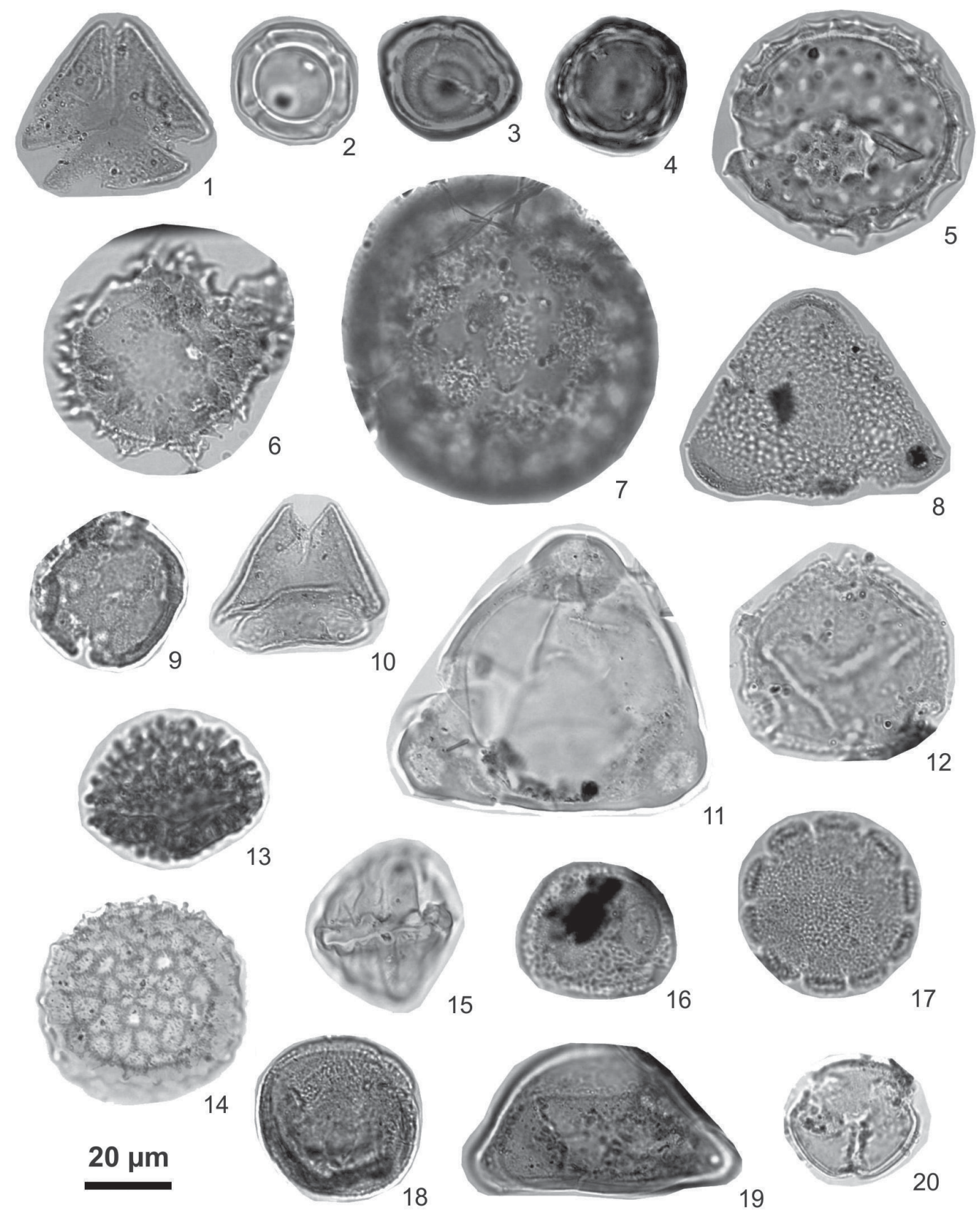

Figure 3. Photomicrographs of gymnosperm and angiosperm pollen grains registered in core BU-91-GL-05 (magnification, $\geq 1000 \times$ ): 1-Type 27 Struthanthus; 2-Type 28 Tetrapteris; 3-Type 29 Heteropteris; 4-Type 30 Peixotoa; 5-Type 31 Abutilon; 6-Type 32 Sida; 7 -Type 33 Hibiscus; 8-Type 34 Pseudobombax; 9-Type 35 Meliaceae; 10-Type 36 Myrtaceae; 11 -Type 37 Fuchsia; 12-Type 38 Ludwigia; 13-Type 39 Passiflora; 14-Type 40 Polygonum; 15-Type 41 Polygala; 16-Type 42 Faramea; 17-Type 43 Borreria; 18-Type 44 Psychotria; 19-Type 45 Sapindaceae; 20-Type 46 Solanaceae. Scale: $20 \mu \mathrm{m}$.

Bauhinia, Pseudobombax, Abutilon, Faramea, Apocynaceae, Tabebuia, Ilex, Tetrapteris, Psychotria, Adenocalymma and Convolvulaceae); and periodically flooded areas, including some restingas, marshes, river banks and lakeshores (Poaceae, Myrtacae, Hibiscus, Adenocalymma, Arrabidaea, Ludwigia, Utricularia, Peixotoa, Desmodium and Inga).

The identity of the pollen grains from the one Poaceae family described in this paper was verified through assessment of their morphological features. However, it is not yet possible to distinguish all taxa down to the genus level. Coincidentally, a flora identified in restingas of the northern coast of Rio de Janeiro indicate that genera belonging to Poaceae occur in coastal environments, such as sandbanks (Andropogon, Eragrostis, Gymnopogon, Rhynchelytrum and Sporobolus); in flooded areas and in the transition between sandbanks and marshlands (Dac- 
tyloctenium and Paspalum); and in restinga (Stenotaphrum and Streptochaeta).

In floristic studies of the areas surrounding the lagoons in the Grussaí-Iquipari drainage basin, near the municipality of São de João Barra, in northern Rio de Janeiro, Assumpção \& Nascimento (2000) recorded four vegetation formations that also correspond to pollen types deposited in the Campos Basin during the Pleistocene-Holocene: beach grass/ marsh grass formation (Poaceae, Alternanthera, Blutaparon and Borreria); beach grass with shrubs (Myrtaceae, Poaceae, Desmodium, Polygala and Borreria); Clusia-dominated formations (Myrtaceae, Meliaceae, Tournefortia, Poaceae, Inga and Sapindaceae); and restinga (Bignoniaceae, Arrabidaea, Pseudobombax, Garcinia, Inga, Heteropteris, Myrtaceae and Sapindaceae). The pollen grains described are also related to the shrub-arboreal component in Coastal Woodlands National Park, near Lake Comprida and Lake Cabiúnas, along the northern coast of Rio de Janeiro. Pimentel et al. (2007) analyzed the taxa richness and diversity among beaches, dunes and Clusia-dominated formations. The taxa found were: Croton, Garcinia, Heteropteris, Ilex, Inga, Meliaceae, Tribe Vernonieae (Vernonia) and Myrtaceae.

\section{Herbaceous vegetation}

The Poaceae pollen grains identified in the present study are also related to restinga vegetation in open upper montane areas (Rizzini 1997) or grassy woodlands that occupy wetlands, near rivers and lakes and in association with Asteraceae and Cyperaceae (IBGE 1992). Taxa commonly associated with herbaceous vegetation include Poaceae, Amaranthus, Alternanthera, Gomphrena, Chenopodium, Borreria, Polygala, Utricularia, Asteraceae, Peixotoa, Desmodium, Ericaceae, Adenocalymma and Convolvulaceae. This association is characteristic of xerophytic and hygrophytic vegetation, reflecting grassland or open areas with some riparian or lacustrine influence.

The results of a palynological analysis carried out by Behling et al. (2002) in the Campos Basin suggest that grass-dominated ecosystems composed most of the landscape during glacial cycles in southeastern Brazil, thus reflecting a colder, drier climate in northern Rio de Janeiro. Palynological data from the Albacora Slope sediments, in the same basin, indicate the presence of grasses in the glacial and interglacial cycles (Freitas 2005). The low frequency of grasses in the samples analyzed $(<10 \%)$ is more likely to be due to changes in sea level than to be a response to significant climatic changes in the northern region of the state of Rio de Janeiro during the Pleistocene-Holocene.

\section{Flooded coastal plain environments}

Coastal plain environments are home to a variety of habitats, including wetlands; fresh water lakes; brackish and salt water lagoons; flood plains; and partially or periodically flooded forests (MMA 2002). Taxa observed in the present study-herbaceous (Poaceae, Polygonum, Ludwigia, Borreria, Polygala and Utricularia); shrub-arboreal (Vernonia, Stigmaphyllon, Garcinia, Alchornea, Croton, Inga, Desmodium, Pseudobombax, Meliaceae, Tabebuia and Ilex); and lianas (Passiflora, Sapindaceae, Psychotria) -also correspond to vegetation occurring in periodically or permanently flooded areas of the Jurubatiba Coastal Woodlands National Park. These exist in lakes, salt marshes and flooded coastal plains in the northern part of the state of Rio de Janeiro (Costa \& Dias 2001; Bove et al. 2003).

\section{Conclusions}

On the basis of the morphological descriptions and taxonomic identification of pollen grains, we can make some inferences regarding the paleovegetation of continental areas adjacent to the Campos Basin during the Pleistocene-Holocene, thus contributing to the current body of research in the area. The pollen types identified in this study are attributed to the following, as currently found in the north of the state of Rio de Janeiro: dense lowland and montane rain forest; seasonal semideciduous forest, restinga; herbaceous vegetation; and hygrophytes common to wetland environments.

\section{Acknowledgments}

This study received financial support from Petróleo Brasileiro (Petrobras, Brazilian Petroleum; Research Grant no. RH-UC-63.011/2003 to AGF), from the Fundação de Amparo a Pesquisa do Estado do Rio de Janeiro (FAPERJ, Foundation for the Support of Research in the State of Rio de Janeiro; laboratory supplies) and from the Brazilian Conselho Nacional de Desenvolvimento Científico e Tecnológico (CNPq, National Council for Scientific and Technological Development; research grant to VG-E). We are grateful to Dr. João Graciano Mendonça Filho, of the Geology Department of the Universidade Federal do Rio de Janeiro (UFRJ, Federal University of Rio de Janeiro), for providing the core to be analyzed; to the geochemist Taíssa R. Menezes, of the Petrobras Center for Research, for the chemical processing of samples; and to Dr. Elizabete P. Ferreira, of the Biostratigraphy and Applied Paleoecology Sector of the Petrobras Center for Research, and Dr. Mauro B. de Toledo, of the Laboratory of Marine Geology at Fluminense Federal University, for the valuable palynological discussions.

\section{References}

Aguiar, A.C.A.; Gonçalves, V.M.; Gonçalves-Esteves, V.L; Yamamoto, K. 2008. Palynotaxonomy of Brazilian species of Polygala. Botanical Journal of the Linnean Society 157: 609-619.

APG III. Angiosperm Phylogeny Group. 2009. An update of the Angiosperm Phylogeny Group classification for the orders and families of flowering plants: APG III. 161: 105-121. 
Araujo, D.S.D. 2000. Análise florística e fitogeográfica das restingas do Estado do Rio de Janeiro. Tese de Doutorado. Programa de Pós-Graduação em Ecologia, Universidade Federal do Rio de Janeiro, Rio de Janeiro.

Asssumpção, J. \& Nascimento, M.T. 2000. Estrutura e composição florística de quatro formações vegetais de Restinga no complexo lagunar Grussaí/Iquipari, São João da Barra, RJ, Brasil. Acta Botanica Brasilica 14(3): 301-315.

Behling, H.; Arz, H.W.; Pätzold, J.; Wefer, G. 2002. Late Quaternary and Climate dynamics in Southeastern Brazil, inferences from marine slope cores GeoB 3229-2 and GeoB 3202-1. Palaeogeography, Palaeoclimatology, Palaeoecology 179: 227-243.

Bove, C.P.; Gil, A.S.B.; Moreira, C.B. \& Anjos, R.F.B. 2003. Higrófitas fanerogâmicas de ecossistemas aquáticos temporários de planície costeira do Estado do Rio de Janeiro, Brasil. Acta Botanica Brasilica 17(1): 119-135.

Caddah, L.F.G.; Kowsmann, R.O. \& Viana, A.R. 1998. Slope sedimentary facies associated with Pleistocene and Holocene sea-level changes, Campos basin, southeast Brazilian Margin. Sedimentary Geology 115: $159-174$.

Carauta, J.P.P. \& Rocha, E.S.F. 1988. Conservação da flora do trecho fluminense da bacia hidrográfica do rio Paraíba do Sul. Albertoa 11(1): 86-135.

Carrijo, T.T.; Mendonça, C.B.F.; Esteves, R.L.; Gonçalves-Esteves, V.L. 2005. Palinotaxonomia de espécies de Stilpnopappus Mart. Ex DC. e Strophopappus DC. (Compositae). Hoehnea 32(2): 259-268.

Costa, A.F. \& Dias, I.C.A. 2001. Flora do Parque Nacional da Restinga de Jurubatiba e arredores; Rio de Janeiro, Brasil: listagem, florística e fitogeografia: angiospermas, pteridófitas, algas continentais. Rio de Janeiro, Museu Nacional - Série Livros.

Freitas, A.G. 2005. Reconstrução paleoclimática com base na análise de palinomorfos continentais em sedimentos pleistocênicos-holocênicos do talude continental brasileiro de Bacia de Campos, RJ. M.Sc. Dissertation, Universidade Federal do Rio de Janeiro, Rio de Janiero.

Freitas, A.G.; Carvalho, M.A. \& Mendonça Filho, J.G. 2007. Description of bryophyte and fern spores and fresh-water algae of PleistoceneHolocene sediments of Albacora Slope, Campos Basin, RJ, Brazil. Pp. 457-470. In: Carvalho, I.S.; Cassab, R.T.; Schwanke, C.; Carvalho, M.A.; Fernandes, A.C.S.; Rodrigues, M.A.C.; Carvalho, M.S.S.; Arai, M.; Oliveira, M.E.Q. (Eds.) Paleontologia: Cenários de Vida, vol 2. Rio de Janeiro, Interciência.

Garcia, M.J. 1994. Palinologia de turfeiras quaternárias do médio vale do Rio Paraíba do Sul, Estado de São Paulo. Tese de doutorado, Programa de Pós-Graduação submetida ao Instituto de Geociências da Universidade de São Paulo. 3 vols.

Gonçalves-Esteves, V.; Junior, E.F.S. \& Mendonça, C.B.F. 2007. Palinologia de espécies de Malpighiaceae Juss. ocorrentes nas restingas do Estado do Rio de Janeiro. Hoehnea 34(4): 519-529.

Instituto Brasileiro de Geografia e Estatística (IBGE). 1992. Manual técnico da vegetação brasileira. Rio de Janeiro: Fundação Instituto Brasileiro de Geografia e Estatística - IBGE.

Kurtz, B.C. \& Araujo, D.S.D. 2000. Composição florística e estrutura do componente arbóreo de um trecho de Mata Atlântica na Estação Ecológica Estadual do Paraíso, Cachoeiras de Macacu, Rio de Janeiro, Brasil. Rodriguésia 51(78/79): 69-111.

Kurtz, B.C.; Sá, C.F.C. \& Silva, D.O. 2009. Fitossociologia do componente arbustivo-arbóreo de florestas semidecíduas costeiras da região de Emerenças, área de proteção ambiental do Pau Brasil, Armação dos Búzios, Rio de Janeiro, Brasil. Rodriguésia 60(1): 129-146.

Lorscheitter, M.L. 1989. Palinologia de sedimentos quaternários do testemunho T15, Cone do Rio Grande, Atlântico Sul, Brasil. Descrições taxonômicas - parte II. Pesquisas 22: 89-127.

Loueuille, B. Souza-Souza, R.M.B; Abreu, V.H.R.; Mendonça. C.B.F.; Gonçalves-Esteves, V.L. 2012. Pollen morphology of the genus Eremanthus Less. (Vernonieae, Asteraceae). Acta Botanica Brasilica 26: 46-57.

Luz, C.F.P. \& Barth, O.M. 2002. Palinomorfos indicadores de tipos de vegetação em sedimentos holocênicos da Lagoa de Cima, Norte do Estado do Rio de Janeiro, Brasil - Dicotyledoneae. Leandra 15: 11-34.

Luz, C.F.P.; Barth, O.M. \& Silva, C.G. 2006. Dinâmica temporal na lagoa do Campelo, região norte do Estado do Rio de Janeiro baseada em estudos palinológicos. Revista Brasileira de Paleontologia 9: 127-136.
Luz, C.F.P.; Barth, O.M.; Martin, L.; Silva, C.G. \& Turcq, J. 2011. Palynological evidence of the replacement of the hygrophilous Forest by Field vegetation during the last 7,000 years B.P. in the northern coast of Rio de Janeiro, Brazil. Anais da Academia Brasileira de Ciências 83: 939-951.

Medeanic, S.; Costa César, S.B. \& Corrêa, I.C.S. 2006. Os palinomorfos nas reconstruções paleoambientais: Azolla filiculoides Lam. Nos sedimentos holocênicos da planície costeira do Rio Grande do Sul. Gravel 4: 49-61.

Mendonça, C.B. F.; Gonçalves-Esteves, V.L \& Esteves, R.L. 2007a. Palinotaxonomia de espécies de Lepidaploa (Cass.) Cass. (VernoniinaeCompositae) ocorrentes no Sudeste do Brasil. Revista Brasileira de Botânica 30: 69-86.

Mendonça, C.B.F.; Gonçalves-Esteves, V.L.; Souza, M.A.; Esteves, R.L. 2007b. Estudio polínico de Albertinia brasiliensis Spreng., de espécies de Cololobus H. Rob., Cyrtocymura H. Rob. y Dasyanthina serrata (Less.) H. Rob. (Vernoniinae-Compositae) que ocurren el sureste del Brasil. Polibotánica 23: 77-92.

Mendonça, C.B.F.; Gonçalves-Esteves, V.L \& Nunes, A. D. 2009. Palynotaxonomy of Vernonanthura H. Rob. (Vernonieae-Compositae) species from southeast, Brazil. Revista Brasileira de Botânica 32: 625-640.

Mendonça, C.B.F.; Carrijo, T.T. \& Gonçalves-Esteves, V.L. 2010. Lessigianthus $\mathrm{H}$. Rob. (Vernonieae-Asteraceae): generic and infrageneric relationships based oh pollen morphology. Nordic Journal of Botany 28: 1-10.

Mendonça-Filho, J.G.; Carvalho, M.A. \& Menezes, T.R. 2002. Palinofacies. Pp. 20-24. In: Técnicas de procedimentos de trabalho com fósseis e formas modernas comparativas. São Leopoldo, UNISINOS.

Milward-Azevedo, M.A.; Souza, F.C.; Baumgratz, J.F.A.; Gonçalves-Esteves, V.L. 2010. Palinotaxonomia de Passiflora L. subg. Decaloba (DC.) Rchb. (Passifloraceae) no Brasil. Acta Botanica Brasilica 24: 133-145. Milward-Azevedo, M.A.; Gonçalves-Esteves, V.L. \& Baumgratz, J.F.A. 2012. Taxonomic revision of Passiflora L. subgenus Decaloba (DC.) Rchb. (Passifloraceae) in Brazil. Phylotaxa 53: 1-68.

Ministério do Meio Ambiente (MMA). 2002. Avaliações e Ações Prioritárias para a Conservação da Biodiversidade das Zonas Costeira e Marinha. Brasília, Fundação Bio-Rio, SECTAM/PA, IDEMA, SNE, SMA/SP, FEPA H.L.

Montezuma, R.C.M. \& Araujo, D.S.D. 2007. Estrutura da vegetação de uma restinga arbustiva inundável no Parque Nacional da Restinga de Jurubatiba, Rio de Janeiro. Pesquisas, Botânica 58: 157-176.

Moreira, F.F.; Mendonça, C.B.F.; Pereira, J.F. \& Gonçalves-Esteves, V. 2004. Palinotaxonomia de espécies de Apocynaceae ocorrentes na Restinga de Carapebus, Carapebus, RJ. Acta Botanica Brasilica 18(4): 711-721.

Moreno, M.R.; Nascimento, MT. \& Kurtz, B. 2003. Estrutura e composição florística do estrato arbóreo em duas zonas altitudinais na mata atlântica de encosta da região do Imbé, RJ. Acta Botanica Brasilica 17: 371-386.

Nimer, E. 1979. Climatologia do Brasil. V. 4. Rio de Janeiro, Secretaria de Planejamento da Presidência da República. IBGE. Série Recursos Naturais e Meio Ambiente.

Peçanha, A.F.; Esteves, R.L. \& Gonçalves-Esteves, V.L. 2008. Palinotaxonomia de Albertinia brasiliensis Spreng., Eremanthus bicolor (DC.) Baker e Vanillosmopsis erythropappa (DC.) Sch. Bip. (CompositaeVernonieae). Acta Botanica Brasilica 22: 313-321.

Pereira, O.J. \& Araujo, D.S.D. 2000. Análise florística das restingas dos Estados do Rio de Janeiro e Espírito Santo. Pp. 25-63. In: Esteves, F.A. \& Lacerda, L.D. (Eds.) Ecologia de Restingas e Lagoas Costeiras. Macaé, NUPEM/UFRJ.

Pereira, M.C.A.; Cordeiro, S.Z. \& Araujo, D.S.D. 2004. Estrutura do estrato herbáceo na formação aberta de Clusia do Parque Nacional da Restinga de Jurubatiba, RJ, Brasil. Acta Botanica Brasilica 18(3): 677-687.

Pessoa, S.V.A. \& Oliveira, R.R. 2006. Análise estrutural da vegetação arbórea em três fragmentos florestais na reserva biológica de Poço das Antas, Rio de Janeiro, Brasil. Rodriguésia 57(3): 391-411.

Pimentel, M.C.P.; Barros, M.J.; Cirne, P.; Mattos, E.A.; Oliveira, R.C.; Pereira, M.C.A.; Scarano, F.R.; Zaluar, H.L.T. \& Araujo, D.S.D. 2007. Spatial variation in the structure and floristic composition of "restinga" vegetation in southeastern Brazil.Revista brasileira de Botânica 30(3): 543-551. 
Punt, W.; Blackmore, S.; Nilsson, S. \& Thomas, A. 2007. Glossary of pollen and spore terminology. Review of Palealeobotany and Palynology 143: 1-81.

RADAMBRASIL. 1983. Levantamento de Recursos Naturais. Folhas Rio de Janeiro/Vitória (SF.23 / 24).

Ramsey, C.; Buck C.E.; Burr, G.S.; Edwards, R.L.; Friedrich, M.; Grootes, P.M.; Guilderson, T.P.; Hajdas, I.; Heaton, T.J.; Hogg, A.G.; Hughen, K.A.; Kaiser, K.F.; Kromer, B.; McCormac, F.G.; Manning, S.W.; Reimer, R.W.; Richards, D.A.; Southon, J.R.; Talamo, S.; Turney, C.S.M.; Van Der Plicht, J. \& Weyhenmeyer, C.E. 2009. IntCal09 and Marine09 radiocarbon age calibration curves, 0 - 50,000 years cal BP. Radiocarbon 51: 1111-1050.

Rizzini, C.T. 1997. Tratado de Fitogeografia do Brasil: Aspectos sociológicos e florísticos. 2. ed. Rio de Janeiro, Âmbito Cultural Edições Ltda.

Salgado-Labouriau, M.L. 1973. Contribuição à Palinologia dos Cerrados. Rio de Janeiro, Academia Brasileira de Ciências.

Silva, G.C. \& Nascimento, M.T. 2001. Fitossociologia de um remanescente de mata sobre tabuleiros no norte do estado do Rio de Janeiro (Mata do Carvão). Revista Brasileira de Botânica 24(1): 51-62.

Souza, V.C. \& Lorenzi, H. 2008. Botânica Sistemática: guia ilustrado para identificação das famílias de fanerógamas nativas e exóticas no Brasil, baseado na APG II. 2.ed. Nova Odessa, Instituto Plantarum.
Souza, F.C.; Souza, M.A.; Mendonça, C.B.F. \& Gonçalves-Esteves, V.L. 2004. Estudo polínico de espécies de Aeschynomeneae e Phaseoleae (Papilionoideae-Leguminosae Juss.) ocorrentes nas restingas do Estado do Rio de Janeiro. Arquivos do Museu Nacional 62:357-366.

Stuiver, M. \& Reimer, P.J. 1993. Extended ${ }^{14} \mathrm{C}$ data base and revised CALIB $3.0{ }^{14} \mathrm{C}$ age calibration program. Radiocarbon 35: 215-230.

Toledo, M.B.; Barth, O.M.; Silva, C.G. \& Barros, M.A. 2009. Testing multivariate analysis in paleoenvironmental reconstructions using pollen records from lagoa Salgada, NE, Rio de Janeiro State, Brazil. Anais da Academia Brasileira de Ciências 81: 757-768.

Tyson, R.V. 1995. Sedimentary organic matter. Organic fácies and palynofacies. Londres, Chapman \& Hall.

Vicalvi, M.A. 1997. Zoneamento bioestratigráfico e paleoclimático dos sedimentos do Quaternário superior do talude da Bacia de Campos, RJ, Brasil. Boletim de Geociências da Petrobras 11: 132-165.

Vicalvi, M.A. 1999. Zoneamento bioestratigráfico e paleoclimático do Quaternário Superior do Talude da Bacia de Campos e do Platô de São Paulo adjacente, com base em foraminíferos planctônicos. Tese de Doutorado, Instituto de Geociências, Universidade Federal do Rio de Janeiro, Rio de Janeiro. 